\title{
Robust design of all-optical PSK regenerator based on phase sensitive amplification
}

\author{
Radan Slavík $^{1 *}$, Francesca Parmigiani ${ }^{1}$, Joseph Kakande ${ }^{1}$, Mathias Westlund ${ }^{2}$, Mats Sköld ${ }^{2}$, \\ Lars Grüner-Nielsen ${ }^{3}$, Richard Phelan ${ }^{4}$, Periklis Petropoulos ${ }^{1}$, and David J. Richardson ${ }^{1}$ \\ 1. Optoelectronics Research Centre, University of Southampton, Southampton, SO17 1BJ, UK \\ 2. EXFO Sweden AB, Arvid Hedvalls Backe 4, SE-41133 Göteborg, Sweden \\ 3. OFS, Priorparken 680, 2605 Brøndby, Denmark \\ 4. Eblana Photonics, Dublin, Ireland \\ *r.slavik@soton.ac.uk
}

\begin{abstract}
More compact, stable, and efficient configuration of a recently-developed regenerator is presented. The regenerator is assessed at data rates up to $56 \mathrm{Gbit} / \mathrm{s}$ using white phase noise for the first time.

(C2011 Optical Society of America

OCIS codes: (060.2320) Fiber optics amplifiers and oscillators, (190.4410) Nonlinear optics, parametric processes
\end{abstract}

\section{Introduction}

Phase noise introduced during transmission both from optical amplifiers and the nonlinear interactions between channels represents a significant limiting factor to data transmission using (differential) phase-shift keyed (D)PSK, modulation formats [1]. Consequently, there is great interest in developing all-optical regeneration schemes capable of eliminating phase (and ideally additionally amplitude) noise for PSK signals. Phase regeneration can be implemented directly by exploiting the phase-squeezing capability of phase sensitive amplifiers (PSAs) [2], and amplitude regeneration can be simultaneously performed by operating in the saturated regime. In order to achieve PSA, a phase relationship between the PSA pump(s), the signal and any idlers present needs to be maintained, which is challenging in practice. Recently, we demonstrated a scheme in which the carrier from a carrier-less PSK signal is recovered and then used to phase-lock the locally generated pumps with the incoming data prior to a degenerate PSA. In this way, a "black-box" operation was obtained [3]. In this scheme, a fraction of the incoming data stream was tapped off and this tapped signal was used for phase locking of the locally-generated pumps. The pump phase locking building block, however, included a relatively long $(300 \mathrm{~m}$ ) highly nonlinear fiber (HNLF), which made the unit susceptible to acoustic/thermal pick up capable of causing strong variations of the phase (more than 2 ) of the locked pumps with respect to the original data. Although it proved possible to compensate for this using a relatively slow (kHz range) feedback loop driving a piezo-transducer (PZT) in the relatively 'quiet' conditions in our laboratory this would not be practical for real-world deployment.

Here, we propose and experimentally demonstrate a new scheme that strongly reduces the effective fiber length over which acoustic/thermal pickup can influence the performance of the regenerator (potentially reducing it to hundreds of millimeters rather than hundreds of meters). Further, we assess the performance of the device at repetition rates up to $56 \mathrm{Gbit} / \mathrm{s}$ with broadband phase noise applied to the data signal rather than the deterministic phase perturbations at discrete frequencies as we did in our earlier work [3].

\section{Principle of operation and experimental results}

Our setup is shown in Fig. 1. The non-return-to-zero (NRZ)-DPSK, $2^{31}-1$ pseudo-random bit sequence (PRBS) was sent through a phase modulator to add the noise. The modulator was driven by a fast photodiode that was fed by ASE noise. The distorted signal was then launched into the black-box regenerator that was made of polarisation maintaining (PM) components other than the part operating in reflection (where the polarization was inherently preserved as a result of the bidirectional propagation) and the HNLF used for PSA (HNLF 2). Rather than tapping off the data signal for the synchronization of the pumps as previously, the whole data signal was used in the synchronization process. First, the signal was combined with a CW local pump ('Pump 1', its frequency was 200 $\mathrm{GHz}$ apart from the data carrier frequency) using an add-multiplexer (data insertion loss $\sim 0.5 \mathrm{~dB}$ ) and then sent to a PM-HNLF (HNLF 1) to generate an idler wave that was inherently phase-locked to the data and Pump 1. The length, dispersion, nonlinear coefficient and insertion loss of HNLF 1 were $300 \mathrm{~m}, 1.3 \mathrm{ps} / \mathrm{nm} / \mathrm{km}, 10.5 \mathrm{~W}^{-1} \mathrm{~km}^{-1}$ and $0.9 \mathrm{~dB}$, respectively. Note that due to the phase erasure process, the binary data modulation was not transferred to the idler [4]. Then, the three signals (Data, Pump 1, Idler) were demultiplexed in a 4-channel 200-GHz demux (insertion loss $\sim 1 \mathrm{~dB}$ ) placed behind a circulator. A mirror provided retro-reflection in the data path as well in the path for Pump 1 which also included a PZT stretcher $(60 \mathrm{~mm}$ total length, maximum phase change of 5 ) and a variable attenuator. A semiconductor laser [5] which was injection locked to the idler, was used to generate Pump 2 
in the idler path. Pump 2 was thus phase-locked to the idler (and thus also to the data and Pump 1). Note that since the injection locking is a much slower process than four wave mixing (having a (sub)-GHz bandwidth) then any high frequency fluctuations (e.g., bit-to-bit phase variations) present on the original data signal are not transferred onto the output of the slave laser [4]. The operation of the demux in the double-pass (reflection) mode has several advantages: (i) it allows for direct injection locking of the semiconductor laser, (ii) it improves the filtering characteristics of the demux which is essential in the following PSA stage, and (iii) all the components operating in reflection (WDM demux, PZT transducer, attenuator, fiber-coupled mirrors) do not need to be polarization maintaining in order to obtain stable polarization operation. Note that all the optical waves involved in the regeneration process share a common path through the regenerator other than on the output side of the demux. The lengths of fiber at the demux output ports can however be kept short $(<2 \mathrm{~m}$ in this case, although this could be reduced to tens of $\mathrm{cm}$ if needed) and all can be kept in close proximity during packaging to ensure that they experience similar acoustic/thermal pickup further reducing the environmental sensitivity of the system.

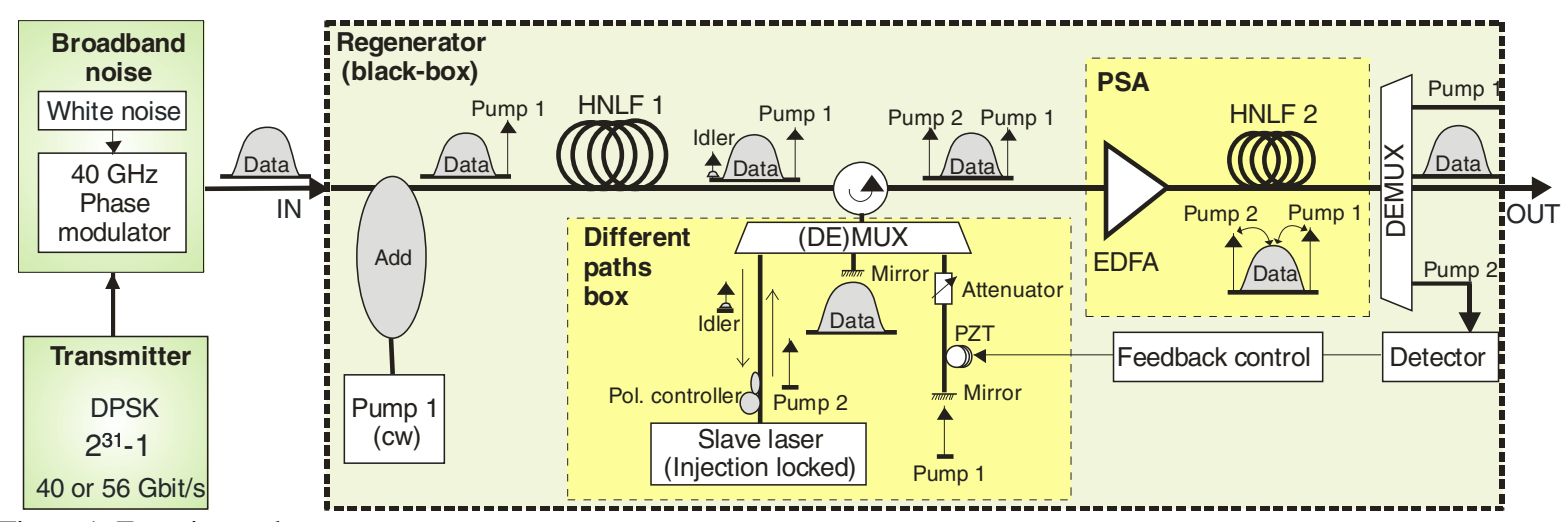

Figure 1: Experimental set-up.

The data stream with the two properly locked pumps was then launched into the PSA which consisted of a highpower EDFA (total power of $29 \mathrm{dBm}$ ) and a 180-m sample of a high-SBS-threshold HNLF [5]. HNLF2 had an alumino-silicate core and had a linear strain gradient along its length (ranging from 400-20 g). The dispersion, polarization mode dispersion, nonlinear coefficient and attenuation of the fiber were $-0.17 \mathrm{ps} / \mathrm{nm} / \mathrm{km}, 0.35 \mathrm{pskm}$, $7.5 \mathrm{~W}^{-1} \mathrm{~km}^{-1}$ and $15 \mathrm{~dB} / \mathrm{km}$, respectively. The relative powers of the pumps and signals were adjusted for optimal regeneration performance (using the attenuator and the bias current of the slave laser in pump paths 1 and 2 respectively). For optimum performance the PSA was operated in deep saturation resulting in a strong variation of pump power at the PSA output as a function of the relative phase between the pump and signal beams. We used this feature to control the PZT fiber stretcher (i.e. the minimum of the pump power corresponds to the maximum PSA gain of the data, which is the correct operation point of the regeneration). No sign of acoustic pick-up was observed in the output signal and only low bandwidth control (Hz-range) was needed to compensate for thermal drift. Just $<5$ of continuous phase adjustment was required to keep the system locked for extended periods of time.

The performance of the regenerator was first studied using an EXFO constellation analyzer (PSO-200) based on all-optical sampling and capable of operation up to $100 \mathrm{Gbaud,} \mathrm{Fig.2.} \mathrm{The} \mathrm{results} \mathrm{at} 40 \mathrm{Gbit} / \mathrm{s}$ and 56 Gbit/s (the maximum data rate of our transmitter) show that the phase noise can be significantly squeezed by the regenerator with negligible induced amplitude noise and also illustrate the bit rate transparency of our regenerator. We measured the bit error ratio (BER) at $40 \mathrm{Gbit} / \mathrm{s}$ (the only modulation speed for which a differential receiver was available) at the input and output of the regenerator for various levels of phase noise, see corresponding measurements in Fig. 3. The receiver consisted of a bandpass filter $(0.5 \mathrm{~nm}$ bandwidth measured at $-0.5 \mathrm{~dB})$, attenuator (the received power was measured at its output), low-noise EDFA, a bandpass filter, a 1-bit delay interferometer (DLI), and a photodetector receiving average power of $7 \mathrm{dBm}$. In Fig. 3a the level of added noise is such that the regenerator is fully capable of restoring the data fidelity despite the fact that the noisy data exhibited an error floor close to $10^{-7}$. In Fig. 3b we show the same data for the noise level used in Fig. 2. In this case, the regenerator fully restores the signal fidelity up to BER $10^{-4}$ (well within the FEC limit), whilst the non-regenerated data suffers from a power penalty of 9 dB. Analyzing Fig. 3 it can be concluded that the regenerator actually improves BER, which is counter-intuitive, as regenerators typically do not improve BER when placed in front of a receiver. This phenomenon is due to a correctable class of errors specific to a 'differential' receiver - e.g., given two consecutive bits with phases $+60^{\circ}$ and $-60^{\circ}$ the signal after a DLI would represent a (differential) phase error of $120^{\circ}$ clearly resulting in an erroneous detection, while the regenerator can restore them both back to $0^{\circ}$ leading to a correctly detected $0^{\circ}$ phase difference. 


\section{OMT2.pdf}

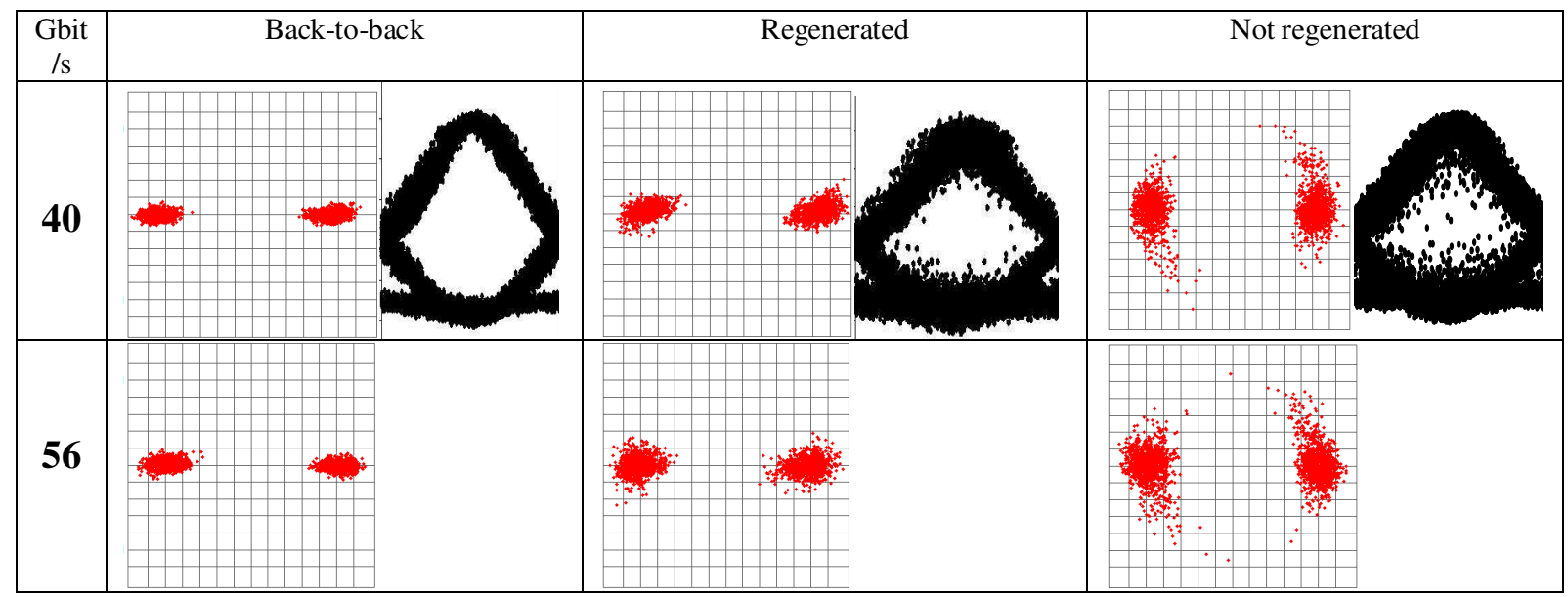

Figure 2: Constellation diagrams for back-to-back and for added noise level: 2 for two data rates and the corresponding demodulated eyes for $40 \mathrm{Gbit} / \mathrm{s}$.
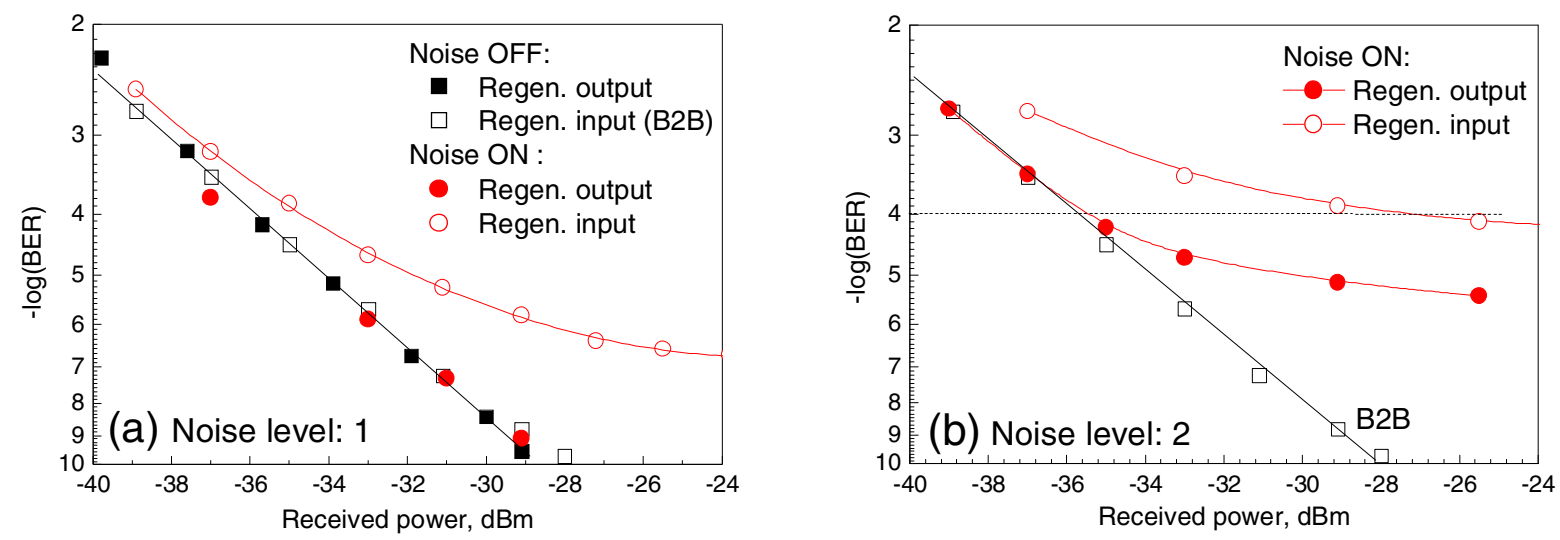

Figure 3: BER curves at the input (open) and output (full) of the regenerator for no added phase noise (black) and two levels of phase noise added (red): (a) noise level 1, (b) noise level: 2 (corresponding to data shown in Fig. 2).

\section{Conclusions}

We have proposed and experimentally demonstrated an improved, compact and more environmentally stable blackbox PSK regenerator using degenerate FWM in a high SBS-threshold highly nonlinear fiber. We tested the regenerator at data rates up to $56 \mathrm{Gbit} / \mathrm{s}$ using - for the first time - white phase noise as would be present in a real fiber network. For moderate levels of phase noise, the regenerator was able to fully restore the signal fidelity (down to a BER of $10^{-9}$ ), while the non-regenerated data showed a floor at a BER of $6 \times 10^{-6}$. For a high level of phase noise, the regenerator fully restored the fidelity of the data (with no power penalty) down to the $10^{-4} \mathrm{BER}$ level, where it gave sensitivity improvement of $9 \mathrm{~dB}$ as compared to the detection of the non-regenerated data.

This research has received funding from the European Communities Seventh Framew ork Programme FP/2007-2013 under grant agreement 224547 (PHASORS).

\section{References}

[1] J. P. Gordon, and L. F. Mollenauer, "Phase noise in photonic communications systems using linear amplifiers," Opt. Lett. 15(23), 1351-1353 (1990).

[2] K. Croussore, et al., "Demonstration of phase-regeneration of DPSK signals based on phase-sensitive amplification," Opt. Express, 13, 39453950 (2005).

[3] R. Slavík et al, “All-optical phase and amplitude regenerator for next-generation telecommunications systems," Nature Photonics 4 , 690695 (2010).

[4] R. Weerasuriya, et al., "Generation of frequency symmetric signals from a BPSK input for Phase Sensitive Amplification", OWT6, paper 1928, OFC 2010.

[5] R. Phelan, B. Kelly, J. O'Carroll, C. Herbert, A. Duke and J. O'Gorman, “- 40 oC < T < 95 oC mode-hop free operation of an uncooled [5] AlGaInAs-MQW discrete-mode laser diode with emission at = 1.3 m.” Electron. Lett. 45, 43-45 (2009).

[6] L. Grüner-Nielsen et al., "A Silica Based Highly Nonlinear Fibre with Improved Threshold for Stimulated Brillouin Scattering," paper Tu.4.D.3, ECOC 2010 